\title{
Descripción de un caso de Fiebre Mediterránea Familiar
}

\author{
Family Mediterranean Fever: a case report
}

\author{
Beatriz Buño Ramilo, Marcos Daniel Montero González, María de Carmen Vázquez Friol, María Dolores Rivera Mosquera \\ Servicio de M. Interna C.H.U. Ferrol.
}

\section{RESUMEN}

La Fiebre Mediterránea Familiar (FMF) es un trastorno autoinflamatorio hereditario de herencia autosómica recesiva, producida por mutaciones en el gen MEFV. La aparición de la enfermedad se produce antes de los 30 años de edad y se caracteriza por ataques recurrentes de fiebre y serositis con una duración de 1-4 días y de curación espontánea.

Presentamos el caso de una familia que cumple criterios clínicos de FMF y presenta únicamente una mutación heterocigota en el gen MEFV lo cual viene a corroborar la posibilidad de un patrón de expresividad dominante en la FMF.

Palabras clave: Fiebre mediterránea familiar, enfermedades autoinflamatorias hereditarias, gen MEFV.

\section{CASO CLÍNICO}

Paciente de 32 años que refiere una historia desde la infancia y hasta la adolescencia consistente en fiebre y amigdalitis de repetición sin respuesta a tratamiento antibiótico. Desde la adolescencia relata dolor osteoarticular sin fiebre, dolor abdominal ni pleurítico. Su hijo de 2 años inicia clínica consistente en episodios febriles, amigdalitis y crisis de dolor abdominal recurrentes y autolimitados con marcadísima elevación de reactantes de fase aguda. En uno de estos episodios se llegó a realizar laparotomía sin hallazgos. Con estos datos se establece el diagnóstico de fiebre mediterránea familiar en base a criterios clínicos (2 criterios mayores) y a la presencia de la mutación E230K en heterocigosis. En base a ello se remite a la paciente para evaluación confirmándose la presencia en heterocigosis de la mutación detectada en su hijo. Desde el punto de vista analítico, coincidiendo con periodos de dolor dorsolumbar, presenta una leve elevación de reactantes de fase aguda y valores también ligeramente incrementados de amiloide sérico con función renal preservada. Las pruebas de imagen a este nivel y los estudios autoinmunes no presentan hallazgos patológicos. Tras el inicio del tratamiento con colchicina evoluciona de forma favorable con desaparición de los dolores osteomusculares que presentaba desde la adolescencia y normalización del amiloide sérico. Al realizar el estudio familiar se objetiva en la madre de la paciente la mutación identificada en ella y en su hijo también en heterocigosis (Figura 1). En este caso la clínica fundamental eran dolores osteomusculares de larga evolución sin otra sintomatología asociada. Desde el punto vista analítico no presentaba alteraciones. A pesar de ello se inició el tratamiento con Colchicina con mala tolerancia por 10 que se ha suspendido.

\begin{abstract}
Familial Mediterranean Fever (FMF) is a hereditary autoinflammatory disorder with an autosomal recessive inheritance pattern, due to mutations in the MEFV gene. The onset of the disease occurs before the age of 30 and is characterized by recurrent attacks of fever and serositis lasting 1-4 days and spontaneously healing.

We present the case of a family that meets clinical criteria for FMF and presents only one heterozygous mutation in the MEFV gene, which corroborates the possibility of a dominant expressiveness pattern in FMF.
\end{abstract}

Keywords: familial mediterranean fever, hereditary autoinflammatory diseases, MEFV gene

\section{DISCUSIÓN}

La Fiebre Mediterránea Familiar (FMF) es un trastorno autoinflamatorio hereditario de herencia autosómica recesiva, producida por mutaciones en el gen MEFV que se encuentra en el cromosoma 16. Es más frecuente entre poblaciones mediterráneas, judíos del norte de África (no ashkenazies), armenios, turcos y árabes, llegando la frecuencia de enfermos en algunas de estas poblaciones a 1/200 individuos. Sin embargo, en los últimos años se han reportado más casos en países no relacionados con esta área'.

Desde el punto de vista clínico la aparición de la enfermedad se produce antes de los 30 años de edad². Cuanto más temprano aparece, más grave es el fenotipo. La FMF puede dividirse en 2 tipos. El tipo 1 se caracteriza por ataques recurrentes de fiebre

Figura 1. Árbol genealógico familiar de la paciente índice

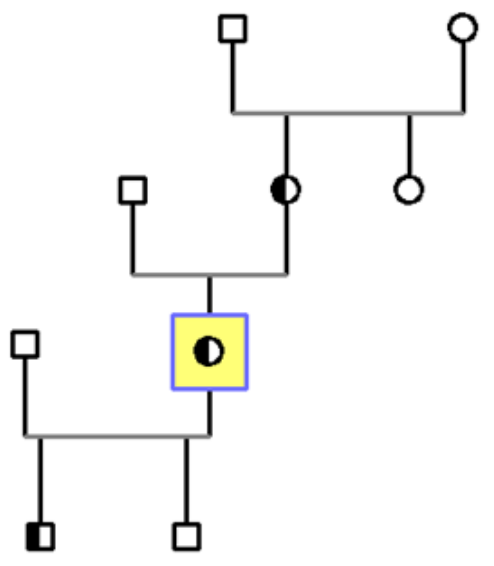


Tabla 1. Características clínicas de los episodios agudos de la fiebre mediterránea familiar.

\begin{tabular}{|l|l|c|}
\hline LOCALIZACIÓN & FORMA CLINICA & $\begin{array}{c}\text { PREVALENCIA } \\
(\%)\end{array}$ \\
\hline Fiebre & Entre $38^{\circ} \mathrm{C}$ y $40^{\circ} \mathrm{C}$ & 99 \\
Abdominal & Peritonitis generalizada & 95 \\
Articular & Monoartritis de grandes & 75 \\
& articulaciones en MMII & 40 \\
Tórax & Pleuritis unilateral & $<1$ \\
& Pericarditis & $<5$ \\
Escroto & Inflamación túnica vaginal unilateral & $<1$ \\
Músculo & Mialgia de uno o varios grupos & $<5$ \\
& musculares & \\
\hline
\end{tabular}

Tabla 2. Criterios clínicos de Tel-Hashomer.

\begin{tabular}{|l|l|l|}
\hline Mayores & Menores & Criterios de apoyo \\
\hline Crisis típicas*: & 1-3 crisis & 1.Historia familiar de FMF \\
Peritonitis & incompletas & 2.Origen étnico asociado \\
(generalizada) & involucrando 1 & a FMF \\
Pleuritis (unilateral) & o más de los & 3.Edad <20 años al inicio \\
o pericarditis & siguientes & de la enfermedad \\
Monoartritis & Abdomen & 4.De la 4 a la 6: \\
(cadera, rodilla, & Tórax & características de los \\
tobillo) & Articulaciones & ataques: \\
Fiebre aislada & Dolor en & 4. Graves: requieren \\
& piernas con el & reposo en cama \\
& esfuerzo & 5. Remisión espontánea \\
& Respuesta & 6. Intervalo libre de \\
& favorable a & síntomas \\
& colchicina & 7. Reactantes de fase \\
& & aguda elevados de forma \\
& transitoria \\
& & 8. Hematuria/proteinuria \\
& episódica \\
& & 9. Laparotomía \\
& sin hallazgos 0 \\
& apendicectomía blanca \\
& 10. Padres cosanguíneos \\
& & \\
& &
\end{tabular}

Para el diagnóstico se requieren $\geq 1$ criterio mayor $0 \geq 2$ criterios menores, $0 \geq 1$ criterio menor más $\geq 5$ criterios de apoyo $0 \geq 1$ criterio menor más $\geq 4$ criterios de apoyo entre los 5 primeros

FMF: fiebre mediterránea familiar; SAA: proteína sérica amiloide

*Crisis típicas: recurrentes ( $\geq 3$ del mismo tipo); febriles ( ${ }^{\mathrm{T}}$ rectal $\geq 38^{\circ} \mathrm{C}$ ) y cortas (duración entre 12 horas y 3 días) y serositis con una duración de 1-4 días y de curación espontánea. El estrés, la exposición al frío, comidas ricas en grasas, infecciones, ciertas drogas y los ciclos menstruales son posibles desencadenantes de los ataques. Los síntomas leves (mialgia, dolor de cabeza, náusea, disnea, artralgia, dolor de espalda baja, astenia y ansiedad) preceden a los ataques al menos unas 17 h. Los ataques se manifiestan por fiebre con duración de 12-72 horas y sin respuesta a antibióticos, dolor abdominal difuso 0 localizado, estreñimiento (diarrea en niños), artralgia, artritis y a veces dolor en el pecho causado por un cuadro de pleuritis $\mathrm{y} / 0$ pericarditis. En el 7-40\% de los pacientes se dan manifestaciones cutáneas. La presencia de otros síntomas es variable, entre los que destacan la esplenomegalia, mialgias, inflamación escrotal aguda, cefalea o determinadas formas de vasculitis (Tabla 1). Los pacientes son asintomáticos en los periodos intercrisis. La amiloidosis tipo AA puede ser una complicación tardía de la enfermedad ${ }^{3}$. El tipo 2 presenta amiloidosis como la primera y única manifestación de la enfermedad.

El diagnóstico es clínico, y se basa en los criterios clínicos Tel-Hashomer (4) (Tabla 2).

Para un diagnóstico definitivo deben existir al menos dos criterios mayores, 0 un criterio mayor y dos criterios menores. Para un diagnóstico probable, se necesita un criterio mayor y un menor.

Se han propuesto nuevos criterios clasificatorios que requieren la presencia de mutaciones confirmatorias (homocigotas 0 heterocigotas compuestas) en el gen MEFV y al menos uno de los 4 criterios mayores ${ }^{5}$. En el caso de no presentar mutaciones confirmatorias, esto es, mutaciones aisladas 0 heterocigotas, los pacientes deben presentar al menos dos criterios mayores. Estos criterios clasificatorios se recomiendan para la inclusión de paciente en ensayos clínicos pero no deben ser utilizados como criterios diagnósticos.

De este modo, los estudios genéticos se utilizan para confirmar el diagnóstico de FMF pero también para excluir otros síndromes febriles hereditarios.

El gen MEFV codifica la proteína pirina (o marenostrina) de 781 aminoácidos. Park el al. (2016) demostraron que la proteína forma un inflamosoma cuando está mutada en respuesta a la modificación bacteriana de la GTPasa RhoA ${ }^{6}$. Este gen tiene más de 300 variantes que se han asociado al fenotipo de FMF, siendo la más frecuentemente observada en los pacientes sintomáticos la variante p.(Met694Val) en el exón 10, que se asocia a un fenotipo más severo7.

La FMF se trata con colchicina, que reduce la frecuencia y la severidad de los síntomas y previene el desarrollo de amiloidosis. En el 5-10\% de los pacientes con FMF la colchicina no es eficaz para prevenir las crisis. Otro 5-10\% de los pacientes son intolerantes a las dosis efectivas y experimentan efectos secundarios graves. En los últimos años, se han realizado estudios de eficacia de otros medicamentos sobre los pacientes resistentes al tratamiento con colchicina. Gül et al. (2015) observaron que Canakinumab fue eficaz para controlar la recurrencia del ataque en pacientes con FMF resistente a 
la colchicina ${ }^{8}$. Van der Hilst et al. (2016) determinaron que la terapia anti IL-1 parece ser una alternativa segura y eficaz para los pacientes con FMF que no responden o no pueden tolerar la colchicina.Ben-Zvi et al. (2017) realizaron un ensayo controlado aleatorio para un tratamiento con Anakinra y observaron mejoría en la sintomatología de los pacientes, por lo que también parece ser un tratamiento eficaz y seguro para la FMF resistente a la colchicina ${ }^{9}$.

Presentamos el caso de una familia sin historia de cosanguinidad ni antecesores procedentes de la cuenca mediterránea portadora de la mutación E230K en heterocigosis. La FMF es habitualmente considerada una enfermedad autosómica recesiva y los pacientes sintomáticos presentan mutaciones patogénicas bialélicas en el gen MEFV localizado en el brazo corto del cromosoma 16. EL $75 \%$ de los casos típicos en armenios, árabes, judíos y turcos presenta una de las estas cinco mutaciones: V726A, M694V, M694I, M680I y E148Q siendo la M694V la más frecuente. Sin embargo, aproximadamente del 10 al $20 \%$ de los pacientes que cumplen criterios diagnósticos para FMF no presenta mutaciones en el gen MEFV10,11.

Se ha encontrado expresión fenotípica en un porcentaje significativo de pacientes que presentan únicamente una mutación en el gen MEFV. El hecho de que estos pacientes con FMF presenten una única mutación sugiere que la enfermedad se podría transmitir también con un patrón de expresividad dominante ${ }^{12}$. Varios trabajos describen una transmisión dominante entre pacientes con mutaciones específicas como la M694VDel, 0 las mutaciones missense H478Y y T577N $\mathrm{N}^{13.14}$. La delección puede causar un serio defecto en la codificación de la proteína pirina llevando a una expresión completa de la enfermedad.

Sin embargo no hay una explicación definitiva para la presencia de FMF en aquellos pacientes portadores de otras mutaciones missense en heterocigosis. Dado que más del 95\% de los portadores de una mutación en heterocigosis en el gen MEFV están asintomáticos se postula que existen factores genéticos adicionales y modificadores ambientales que pueden influir en la expresión fenotípica de la enfermedad. Un estudio que utilizó una aproximación estadística para estimar la contribución de la heterocigosidad a la prevalencia de la de la enfermedad realizó una comparación del genotipo en hermanos de 63 familias con FMF y el estudio genotípico en 557 pacientes de poblaciones mediterráneas. Este estudio demostró que la heterocigosidad no es reponsable de la transmisión mendeliana clásica de la FMF pero constituye un factor de riesgo para desarrollarla con un riesgo seis a ocho veces mayor que en no portadores de la mutación en el gen MEFV ${ }^{15}$.

La familia que presentamos cumple criterios clínicos de FMF y presenta únicamente una mutación heterocigota en el gen MEFV lo cual viene a corroborar la posibilidad de un patrón de expresividad dominante en la FMF.

\section{BIBLIOGRAFÍA}

1. Ben-Chetrit E, Touitou I. Familial mediterranean Fever in the world. Arthritis Rheum. 2009 0ct;61(10):1447-53.

2. Sohar E, Gafni J, Pras M, Heller H Familial Mediterranean fever. A survey of 470 cases and review of the literatura. Am J Med. 1967 Aug:43(2):227.

3. Van der Hilst JC, Simon A, Drenth JP. Hereditary periodic fever and reactive amyloidosis. Clin Exp Med. 2005 0ct;5(3):87.

4. Livneh A, Langevitz P, Zemer D, Zaks N, Kees S, Lidar T, Migdal A, Padeh S, Pras $M$ Criteria for the diagnosis of familial Mediterranean fever.nArthritis Rheum. 1997 Oct; $40(10): 1879$.

5. Gattorno M, Hofer M, Federici S, Vanoni F, Bovis F, Aksentijevich I, et al. Eurofever Registry and the Paediatric Rheumatology International Trials Organisation (PRINTO). Classification criteria for autoinflammatory recurrent fevers. Ann Rheum Dis. 2019 Aug:78(8):1025-1032.

6. Park YH, Wood G, Kastner DL, Chae JJ. Pyrin inflammasome activation and RhoA signaling in the autoinflammatory diseases FMF and HIDS. Nat Immunol. 2016 Aug;17(8):914-21

7. Debeljak M, Toplak N, Abazi N, Szabados B, Mulaosmanovi囚 V, Radovi囚 J, et al. The carrier rate and spectrum of MEFV gene mutations in central and southeastern European populations. Clin Exp Rheumatol. 2015 Nov-Dec;33(6 Suppl 94):S19-23

8. Gül, et al.Efficacy and safety of canakinumab in adolescents and adults with colchicine-resistant familial Mediterranean fever. Arthritis Res Ther. 2015 Sep; 17(1): 243

9. Ben-Zvi, et al. Anakinra for Colchicine-Resistant Familial Mediterranean Fever: A Randomized, Double-Blind, Placebo-Controlled Trial. Arthritis Rheumatol. 2017 Apr;69(4):854-862.

10. Booty MG, Chae JJ, Masters SL, Remmers EF, Barham B, Le JM, Barron KS, Holland SM, Kastner DL, Aksentijevich Familial Mediterranean fever with a single MEFV mutation: where is the second hit? Arthritis Rheum. 2009 Jun;60(6):1851.

11. Touitou I. The spectrum of Familial Mediterranean Fever (FMF) mutations. Eur $\mathrm{J}$ Hum Genet. 2001 Jul;9(7):473

12. Padeh $S$, et al. Clinical and diagnostic value of genetic testing in 216 Israeli children with Familial Mediterranean fever. J Rheumatol. 2003 Jan;30(1):185

13. Marek-Yagel D, et al. Clinical disease among patients heterozygous for familial Mediterranean fever. Arthritis Rheum. 2009 Jun;60(6):1862.

14. Rowczenio DM, Autosomal dominant familial Mediterranean fever in Northern European Caucasians associated with deletion of p.M694 residue-a case series and genetic exploration. Rheumatology (0xford). 2017 Feb;56(2):209.

15. Aldea A, et al. Severe autosomal-dominant periodic inflammatory disorder with renal AA amyloidosis and colchicine resistance associated to the MEFV H478Y variant in a Spanish kindred: an unusual familial Mediterranean fever phenotype or another MEFV-associated periodic inflammatory disorder? Am J Med Genet A. 2004 Jan;124A(1):67. 